\title{
In vitro Comparison of Disk Diffusion and Agar Dilution Antibiotic Susceptibility Test Methods for Neisseria gonorrhoeae
}

\author{
Marta C de Castillo ${ }^{+}$, Olga A de Saab, Olga M de Nader, \\ Aída P de Ruiz Holgado
}

Instituto de Microbiología “Luis C. Verna”, Universidad Nacional de Tucumán, Ayacuco 491, Tucumán, Argentina

At present, most Neisseria gonorrhoeae testing is done with $\beta$-lactamase and agar dilution tests with common therapeutic agents. Generally, in bacteriological diagnosis laboratories in Argentina, study of antibiotic susceptibility of N.gonorrhoeae is based on $\beta$-lactamase determination and agar dilution method with common therapeutic agents. The National Committee for Clinical Laboratory Standards (NCCLS) has recently described a disk diffusion test that produces results comparable to the reference agar dilution method for antibiotic susceptibility of N.gonorrhoeae, using a dispersion diagram for analyzing the correlation between both techniques. We obtained 57 gonococcal isolates from patients attending a clinic for sexually transmitted diseases in Tucumán, Argentina. Antibiotic susceptibility tests using agar dilution and disk diffusion techniques were compared. The established NCCLS interpretive criteria for both susceptibility methods appeared to be applicable to domestic gonococcal strains. The correlation between the MIC's and the zones of inhibition was studied for penicillin, ampicillin, cefoxitin, spectinomycin, cefotaxime, cephaloridine, cephalexin, tetracycline, norfloxacin and kanamycin. Dispersion diagrams showed a high correlation between both methods.

Key words: Neisseria gonorrhoeae - antimicrobial susceptibility - disk diffusion - agar dilution

The ability of bacteria to develop resistance to antimicrobial agents has become a significant problem in the treatment and control of communicable sexually transmitted diseases (Perine et al. 1977, Thornsberry et al. 1977, Ashford et al. 1981, Knapp et al. 1987, CDC 1987, Heritage \& Hawkey 1988). This is particularly true for Neisseria gonorrhoeae, which has evolved a number of different mechanisms to resist antimicrobial agents. These mechanisms are plasmid or chromosome encoded. The emergence of penicillinase-producing $N$. gonorrhoeae (PPNG) in 1976 has led to widespread high-level penicillin resistance (Ashford et al. 1976, CDC 1976, Phillips 1976, Siegel et al. 1978). Highlevel tetracycline resistance (MIC ${ }^{3} 16 \mathrm{mg} / \mathrm{ml}$ ) was reported for $N$. gonorrhoeae in 1985 by the Centers for Disease Control (CDC 1985). Spectinomycin resistance has also been reported for many strains of $N$. gonorrhoeae (Thornsberry et al. 1977, Ashford et al. 1981). Gonococcal resistances have commonly been reported to originate in southeast Asia and Western Africa (Phillips 1976).

${ }^{+}$Corresponding author. Fax: +54-81-24.8169

Received 10 October 1997

Accepted 8 April 1998
There is an immediate need to simplify and standardize the in vitro antimicrobial susceptibility testing of $N$. gonorrhoeae, because of the frequency of isolation and the increased levels of antimicrobial resistance seen with these strains. Antimicrobial susceptibility testing of $N$. gonorrhoeae in most clinical microbiology laboratories in Argentina consists of the B-lactamase test and a $10-\mathrm{U}$ penicillin disk diffusion test, both used for the detection of penicillinase-producing N. gonorrhoeae (Phillips 1976, NCCLS 1990a). Alternative methods, such as agar dilution antimicrobial testing (NCCLS 1990b), are generally laborious and not practical for routine use. The development of a standardized agar diffusion method for these tests is practical and allows fast and reproducible results for clinical microbiology laboratories if standards are observed (Jones et al. 1990a,b). The purpose of this study was to evaluate the agar disk diffusion technique, comparing it with the agar dilution method proposed by the National Committee for Clinical Laboratory Standard (NCCLS), using strains of N. gonorrhoeae, isolated in Tucumán, Argentina.

\section{MATERIALS AND METHODS}

Bacterial strains - Two gonococcal control strains (ATCC 49226: WHO A and WHO C) were selected for susceptibility to penicillin (WHO A: MIC $0.5 \mathrm{mg} / \mathrm{ml}$ and zone diameter $31.1 \mathrm{~mm}$; WHO C: MIC $25 \mathrm{mg} / \mathrm{ml}$ and zone diameter $10.6 \mathrm{~mm}$ ). 
Besides, 57 N.gonorrhoeae strains, isolated from samples from patients attending a clinic in Tucumán between 1990 and 1991, were studied.

Antimicrobial agents - Antimicrobial agents used for the agar dilution studies were penicillin, ampicillin, tetracycline, cefotaxime, norfloxacin, cefoxitin, spectinomycin, cephaloridine, cephalexin and kanamycin, which were provided by the Microbiology Institute "Carlos G. Malbran”, as dry experimental substances with known capacity.

The antimicrobial agent-containing disks included penicillin $(10 \mathrm{U})$, tetracycline $(30 \mathrm{mg})$, cefotaxime $(30 \mathrm{mg})$ norfloxacin $(10 \mathrm{mg})$, spectinomycin $(100 \mathrm{mg})$, cefoxitin $(30 \mathrm{mg})$, ampicillin (10 mg), cephaloridine (30 mg), cephalexin $(30 \mathrm{mg})$ and kanamycin $(30 \mathrm{mg})$. All drug solutions were prepared and stored according to the manufacturers' instructions. All disks were provided by the Microbiology Institute "Carlos G. Malbran" stored in desiccated storage units at $4^{\circ} \mathrm{C}$.

The antimicrobial agents tested, their agar diffusion breakpoints and their MIC limits are shown in Tables I and II. Values are according to the NCCLS (NCCLS 1990a,b).

Laboratory identification of $N$. gonorrhoeae All specimens were obtained with Dacron swabs from male urethral samples. The specimens were immediately plated onto GC-agar (Difco Laboratories, Detroit, $\mathrm{Mi}$ ) plates and incubated at $35^{\circ} \mathrm{C}$ in a candle jar. The isolates were classified as Neisseria spp. by the identification of gram-negative diplococci from the urethral swab, characteristic colony morphology, and an oxidase-positive test. All presumptive gonococcal isolates were frozen at $-70^{\circ} \mathrm{C}$ in tryptic soy broth with $25 \%$ glycerol. The isolates were later plated onto GC-agar plates supplemented with $1 \%$ Iso Vitalex (Becton Dickinson) and incubated for $24 \mathrm{hr}$ at $37^{\circ} \mathrm{C}$ under an atmosphere of 5 to $8 \% \mathrm{CO}_{2}$.

The organisms were harvested and retested for diplococcal morphology and the production of oxidase. All strains were confirmed with a $N$. gonorrhoeae-specific monoclonal antibody assay (Phadebact Monoclonal GC OMNI Test 50; Remel, Lenexa, Ka). All confirmed organisms were tested for the presence of $\beta$-lactamase production with nitrocefin (Cefinase, Glaxo Research Ltd, Greenford, Middlesex, England).

Antimicrobial susceptibility testing - The agar dilution method was performed according to the method established by the NCCLS with GC-agar with $1 \%$ of a defined supplement (NCCLS 1990b). The disk diffusion method was performed with GCagar using 1\% GC supplement (Prepared Media Laboratories), also according to the recommendations of the NCCLS (NCCLS 1990a). The contents of the GC-agar and the supplement have been described previously by Jones et al. (1989). All agar plates (agar dilution and disk diffusion) were incubated at $35^{\circ} \mathrm{C}$ under a 5 to $8 \% \mathrm{CO}_{2}$ atmosphere, and results were read after $24 \mathrm{hr}$.

Data analysis - A dispersion diagram was carried out for each of the antibiotics as to determine the correlation between the MIC and the disk zone diameter susceptibility tests. The correlation coefficient according to Pearson ( $r$ ) is used for measuring the linear correlation between $\log _{2}$ MIC $x$ diameter (Lorian 1986).

\section{RESULTS AND DISCUSSION}

Tables I and II show the distribution of the susceptibility of the studied strains. The isolated strains are highly susceptible to the examined antibiotics except for tetracycline $(12.5 \%$ of the strains showed resistance to this drug).

Two strains out of $57(3.5 \%)$ were resistant to penicillin, ampicillin and cephalosporin of the first generation (cephaloridine-cephalexin).

The distribution of the MIC's and the inhibition halos of the other antibiotics assayed, was similar for both ß-lactamase producing PPNG (plasmid encoded) and non-producing gonococci. Therefore these drugs (tetracycline, spectinomycin, norfloxacin and cephalosporins of the 2nd and 3rd generation) were considered as alternatives for the treatment of infections, which could not be cured with penicillin. The results agreed with those obtained by Mc Cormack (1982).

Jones et al. (1989) determined that $90 \%$ of the laboratories routinely used the $\beta$-lactamase test on all gonococcal strains or selected isolates. The most commonly used methods ( $93 \%$ ) were commercially prepared chromogenic cephalosporin (nitrocefin or PADAC) and acidimetric reagents. PPNG strains were rarely found. Few laboratories performed other susceptibility tests on $N$. gonorrhoeae strains. When another method was used, the disk diffusion procedure was preferred in $86 \%$ of the laboratories. The most-tested anti-gonococcal drugs were penicillin, tetracycline, B-lactamase-stable cephalosporins and spectinomycin.

The standard disk diffusion test allowed to obtain reproducible results, when compared with the MIC method. Gonococci showed $1.7 \%$ more resistance against penicillin, cephalexin and kanamycin with the disk diffusion test than using the MIC method. For tetracycline the difference between both tests was $3.5 \%$ (the disk diffusion test showing more resistance again). Similar results were obtained in assays carried out by Stratton (1984) and by Fekete (1993).

With each type of antibiotic a dispersion diagram was made to analyze the correlation between the susceptibility tests of the diffusion in agar and the MIC (Fig.). The index used for measuring the 
TABLE I

Diffusion method: comparison of the inhibition diameters (zone diameter breakpoint) of 57 strains of Neisseria gonorrhoeae from Tucumán, Argentina; for the tested antibiotics; susceptible strains are in bold

\begin{tabular}{|c|c|c|c|c|c|c|c|c|c|c|c|c|c|c|c|c|c|c|c|c|c|c|c|c|c|c|c|c|c|c|c|c|c|}
\hline \multirow[t]{2}{*}{ Antibiotic } & \multirow{2}{*}{$\begin{array}{r}\begin{array}{r}\text { Disk } \\
\text { load }\end{array} \\
\mu \mathrm{g}\end{array}$} & \multirow{2}{*}{$\begin{array}{c}\text { Range } \\
\mathrm{mm}\end{array}$} & \multicolumn{31}{|c|}{ Zone diameter breakpoint (mm) } \\
\hline & & & 2 & 4 & 6 & 8 & 10 & 12 & 14 & 16 & 18 & 20 & 22 & 24 & 26 & 28 & 30 & 32 & 34 & 363 & 38 & 40 & 42 & 44 & 46 & 48 & 50 & 525 & 54 & 56 & 58 & $60>60$ & Resistance \% \\
\hline Penicillin & 10 & $28-47$ & 2 & & & & & & & & & & & & 1 & 12 & 20 & 8 & & 5 & 5 & 1 & 1 & & 1 & 1 & & & & & & & 5.26 \\
\hline Ampicillin & 10 & $21-40$ & 2 & & & & & & & & & & & 2 & 3 & 5 & 16 & 6 & 3 & 3 & 2 & 3 & 3 & 4 & 2 & 2 & 1 & & & & & & 3.50 \\
\hline Cephaloridine & 30 & $14-18$ & 1 & & & & 1 & & 3 & 16 & 5 & 4 & & & 12 & 9 & 4 & 1 & 1 & & & & & & & & & & & & & & 3.50 \\
\hline Cephalexin & 30 & $15-19$ & 2 & & & & 1 & & & 8 & 2 & 9 & 6 & 6 & 6 & 5 & 6 & 4 & 2 & & & & & & & & & & & & & & 5.26 \\
\hline Cefoxitin & 30 & $23-28$ & & & & & & & & & & & & & & 1 & 1 & 2 & 3 & & & 4 & & & & & 2 & 3 & 5 & 6 & & 208 & 0 \\
\hline Cefotaxime & 30 & $31-35$ & & & & & & & & & & & & & & & & & & 1 & & 1 & & & 1 & & 2 & 2 & & 3 & 8 & 437 & 0 \\
\hline Kanamycin & 30 & $13-18$ & 1 & & & & & & 1 & & 1 & & & & & 2 & 8 & 5 & 5 & 18 & & 1 & & & & & & & & & & & 3.50 \\
\hline Spectinomycin & 100 & $14-20$ & 1 & & & & & & & 2 & 2 & 7 & 11 & 6 & 11 & 15 & 2 & & & & 15 & & & & & & & & & & & & 1.75 \\
\hline Tetracycline & 30 & $30-38$ & & & & & & 1 & & 4 & 2 & & & & & 3 & 18 & 10 & 3 & 7 & 1 & 4 & 2 & & & & & & & 2 & & & 17.50 \\
\hline Norfloxacin & 10 & $14-18$ & & & & & & & & & & & & & & & 1 & 3 & & & 1 & 3 & 11 & 11 & 15 & 10 & 2 & & & & & & 0 \\
\hline
\end{tabular}

TABLE II

Dilution method in vitro susceptibility of 57 strains of Neisseria gonorrhoeae from Tucumán, Argentina; for the tested antibiotics susceptible strains are in bold

\begin{tabular}{|c|c|c|c|c|c|c|c|c|c|c|c|c|c|c|c|c|c|c|c|}
\hline \multirow[b]{2}{*}{ Antibiotic } & \multicolumn{19}{|c|}{$\mathrm{MIC}(\mathrm{mg} / \mathrm{ml})$} \\
\hline & Range & 0,0005 & 0,001 & 0,003 & 0,005 & 0,01 & 0,02 & 0,05 & 0,1 & 0,25 & 0,5 & 1 & 2 & 5 & 10 & 25 & 50 & 100 & 200 \\
\hline Penicillin & $0,06-2$ & & & & & 2 & 2 & 4 & 5 & 8 & 14 & 17 & 3 & & & & & & 2 \\
\hline Ampicillin & $1-4$ & & & & & 1 & 2 & 3 & 4 & 5 & 9 & 26 & 5 & & & & & & 2 \\
\hline Cephaloridine & $4-16$ & & & & & & & & & & 6 & 10 & 11 & 9 & 9 & & 1 & 1 & \\
\hline Cephalexin & $4-16$ & & & & & & & & & & 6 & 11 & 12 & 15 & 11 & 2 & & & \\
\hline Cefoxitin & $2-8$ & & & & & & & 27 & 8 & 6 & 3 & 6 & 5 & 2 & & & & & \\
\hline Cefotaxime & $0,034-2$ & 30 & 8 & 3 & 6 & 2 & 4 & & 1 & 2 & 1 & & & & & & & & \\
\hline Kanamycin & $4-64$ & & & & & & & & & & & 1 & 38 & 15 & & 1 & 1 & 1 & \\
\hline Spectinomycin & $32-128$ & & & & & & & & & & & & 30 & 12 & 10 & 3 & 1 & & 1 \\
\hline Tetracycline & $0,25-2$ & & & & & & & & & 2 & 7 & 9 & 31 & 7 & 1 & & & & \\
\hline Norfloxacin & 4-10 & & & & & 15 & 15 & 16 & 4 & 4 & & & & & & & & & \\
\hline
\end{tabular}



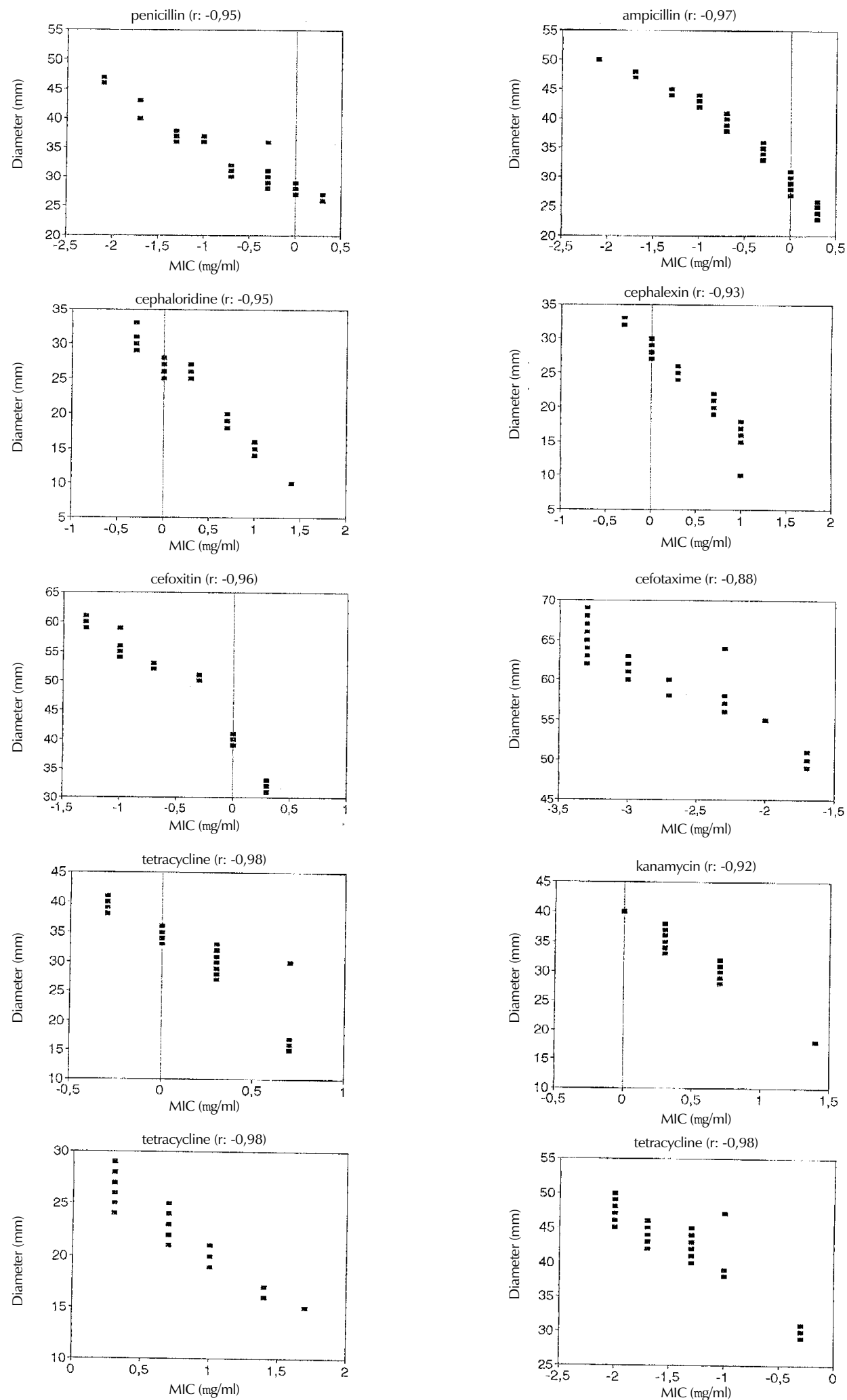

Dispersion diagram between the agar dilution and the agar diffusion methods for 57 Neisseria gonorrhoeae strains from Tucumán, Argentina. Correlation coefficient according to Pearson (r) (see text for details) between both methods is given in brackets for each of the antibiotics studied. 
linear regression analysis grade is the Pearson coefficient (r). A strong correlation between both methods could be observed.

When estimating the sensitivity and the specificity of both methods taking the MIC as the standard value, and classifying the strains in resistant and non-resistant (sensitive) ones, the following results were obtained: a sensitivity of $89 \%$ and a specificity of $91 \%$. Similar results have been found by Jones et al. (1988), obtaining a correlation of $98 \%$ between both methods.

Due to the frequency of gonococcal infections various methods have been developed to study their in vitro susceptibility (Maier et al. 1974, Putnam et al. 1992).

In 1978 the scientific group of the World Health Organization recommended a standard method, with which they achieved to compare the results of different national and international laboratories, as well as the effectiveness of the antimicrobial therapies applied in different geographic areas (WHO 1978).

The results of the disk diffusion test for this microorganism and for the tested antibiotics were perfectly reproducible and comparable with the dilution agar method, MIC, if standardized parameters are observed. Analysis with a dispersion diagram showed a high correlation between both, with a sensitivity of $89 \%$ and a specificity of $91 \%$.

\section{ACKNOWLEDGEMENTS}

To Mr Eric Fengler for language advice and drawing of the graphics.

\section{REFERENCES}

Ashford WA, Golash RG, Hemming VG 1976. Penicillinase-producing Neisseria gonorrhoeae. The Lancet ii: 657-658.

Ashford WA, Adams HJU, Johnson SR, Thornsberry C, Potts DW, English JC, Biddle JW, Jaffe HH 1981. Spectinomycin-resistant penicillinase-producing Neisseria gonorrhoeae. The Lancet 11: 1035-1037.

CDC-Centers for Disease Control 1976. Penicillinaseproducing Neisseria gonorrhoeae-world-wide. Morbid Mortal Weekly Rep 33: 408-410.

CDC-Centers for Disease Control 1985. Tetracyclineresistant Neisseria gonorrhoeae-Georgia, Pennsylvania, New Hampshire. Morbid Mortal Weekly Rep 34: 563-564.

CDC-Centers for Disease Control 1987. Antibiotic-resistant strains of Neisseria gonorrhoeae: policy guidelines for detection, management, and control. Morbid Mortal Weekly Rep 36(Suppl): 1-18.

Fekete T 1993. Antimicrobial susceptibility testing of Neisseria gonorrhoeae and implications for epidemiology and therapy. Clin Microbiol Rev 6: 23-33.

Heritage J, Hawkey PM 1988. Tetracycline-resistant Neisseria gonorrhoeae. J Antimicrob Chemother 22: 575-582.
Jones RN, Edson DC, The Microbiology Resource Committee of the College of American Pathologists 1988. The identification and antimicrobial susceptibility testing of Neisseria gonorrhoeae 1980-1987. Arch Pathol Lab Med 112: 485-488.

Jones RN, Fuchs PC, Washington II JA, Gavan TL, Murray PR, Gerlach EH, Thornsberry C 1990a. Interpretive criteria, quality control guidelines and drug stability studies for susceptibility testing of cefotaxime, cefoxitin, ceftacidime, and cefuroxime against Neisseria gonorrhoeae. Diagn Microbiol Infect Dis 13: 499-507.

Jones RN, Gavan TL, Thornsberry C, Fuchs PC, Gerlach EH, Knapp JS, Murray P, Washington JA 1989. Standardization of disk diffusion and agar dilution susceptibility test for Neisseria gonorrhoeae: interpretive criteria and quality control guidelines for ceftriaxone, penicillin, spectinomycin, and tetracycline. J Clin Microbiol 27: 2758-2766.

Jones RN, Gerlach EH, Koontz FP, Murray PR, Pfaller MA, Washington JA, Erwin ME, Knapp CC 1990b. Development of Neisseria gonorrhoeae in vitro susceptibility test methods for cefixime including quality control guidelines. Diagn Microbiol Infect Dis 14: 383-388.

Knapp JS, Zenilman JM, Biddle JW, Perkins GH, Dewitt WE, Thomas ML 1987. Frequency and distribution in the United States of strains of Neisseria gonorrhoeae with plasmid-mediated, high-level resistance to tetracycline. J Infect Dis 155: 819-822.

Lorian V 1986. Antibiotics in Laboratory Medicine, Williams and Wilkins, Baltimore, p. 281-286.

Maier TW, Beilstein HR, Zubrzycki L 1974. Antibiotic disk susceptibility test with Neisseria gonorrhoeae. Antimicrob Agents Chemother 4(Suppl. 2): 42-43.

Mc Cormack WM 1982. Penicillinase-producing Neisseria gonorrhoeae. A retrospective. $N$ Engl J Med 307: 438-439.

NCCLS-National Committee for Clinical Laboratory Standards 1990a. Approved standard M2-A4. Performance standards for antimicrobic disk susceptibility tests, Villanova, $\mathrm{Pa}$.

NCCLS-National Committee for Clinical Laboratory Standards 1990b. Approved standard M7-A2. Standard methods for dilution antimicrobial susceptibility tests for bacteria that grow aerobically, Villanova, $\mathrm{Pa}$.

Perine PL, Schalla W, Siegel MS 1977. Evidence for two distinct types of penicillinase-producing Neisseria gonorrhoeae. The Lancet ii: 993-995.

Phillips I 1976. Beta-lactamase-producing, penicillinresistant gonococcus. The Lancet ii: 656-657.

Putnam SD, Lavin BS, Stone JR, Oldfield EC, Hooper DG 1992. Evaluation of the standardized disk diffusion and agar dilution antibiotic susceptibility test methods by using strains of Neisseria gonorrhoeae from the United States and Southeast Asia. J Clin Microbiol 30: 974-980.

Siegel MS, Thornsberry C, Biddle JW, O'Mara PR, Perine PL, Weisner PJ 1978. Penicillinase-producing Neisseria gonorrhoeae: results of surveillance 
in the United States. J Infect Dis 137: 170-175.

Stratton CW 1984. Susceptibility testing revisited. Prog Clin Pathol 9: 65-100.

Thornsberry C, Jaffe H, Brown ST, Edwards T, Biddle JW, Thompson SE 1977. Spectinomycin-resistant
Neisseria gonorrhoeae. JAMA 237: 2405-2406.

WHO-World Health Organization 1978. Neisseria gonorrhoeae and gonococcal infection. Report of a WHO Scientific Group. WHO Tech Rep Series 616: 65-91. 